\title{
Review of determinants of national medical leadership development
}

\author{
Wouter Keijser, ${ }^{1,2}$ Max Poorthuis, ${ }^{1}$ Judith Tweedie, ${ }^{3}$ Celeste Wilderom
}

- Additional material is published online only. To view please visit the journal online (http://dx.doi.org/10.1136/ leader-2017-000023).

${ }^{1}$ Faculty of Behavioural, Management and Social Sciences (BMS) Change Management and Organizational Behavior (CMOB), University Twente, Enschede, The Netherlands ${ }^{2}$ Research Department, Dutch Institute of Research and Education in Medical Leadership and Interdisciplinary Teamwork (DIRMInstitute), Utrecht, The Netherlands

${ }^{3}$ Royal College of Physicians, London, UK

\section{Correspondence to}

Wouter Keijser, Faculty of Behavioural, Management and Social Sciences (BMS) Change Management and Organizational Behavior (CMOB), University Twente, AE Enschede 217 7500, The Netherlands:

wouter@keijser.com

Received 7 June 2017

Revised 14 September 2017 Accepted 25 October 2017

Published Online First

18 November 2017

\section{ABSTRACT}

Increasingly, physician engagement in management, quality and innovation is being recognised as vital, requiring 'medical leadership' (ML) competencies. Besides numerous local institutional efforts and despite the high level of autonomy of the medical profession and the education of its members, in some countries, national level activities are focusing on developing $\mathrm{ML}$ competencies to guide physicians in more effectively engaging in these non-medical activities. Up to this date, little is known about effective strategies and tactics for developing ML on a national level. This study investigates existing literature on determinants and interventions for national ML development. We performed a scoping review and subsequent systematic literature review of published reviews, using PubMed, Scopus, Web of Science, Ovid MEDLINE and Science Direct in search for eligible papers between 2011 and 2016. Full-text versions of 43 papers were studied, and a snowballing method was deployed. Data extraction included grounded theory coding, and synthesis of data was done iteratively during data clinics. Analysis of the seven included papers resulted in five discrete categories of determinants of and 10 distinct interventions relevant to national development of ML approaches. None of the papers reported on any specific phasing of national ML development. Our data suggest that local and national level activities in ML development should consider multifaceted and multilevel approaches, taking into account resistance to change and redesign of institutionalised logics that accompany changing positions and reconstruction of professional identities of physicians.

\section{INTRODUCTION}

In line with various transformational activities in healthcare systems across the world, the role of leaders in healthcare changes and innovations, that are advantageous for patients, is increasingly being reported. ${ }^{12}$ The beneficial effect of 'medical leadership' (ML) on healthcare quality as well as on patient and employee satisfaction is well documented, and calls for doctors to engage in effective leadership development are on the rise. ${ }^{3}$ Furthermore, patient safety, one of the cornerstones of high-quality care, has now become a worldwide concern, and it needs stout (medical) leadership engagement. ${ }^{4}$ Also, from an economical perspective, doctors have a great impact on the use of resources; they are being urged to be concerned with effective healthcare resource deployment and distribution in the face of budget constraints and rising costs. ${ }^{5}$ The increasing complexity and interprofessional collaborations that propel task and boundary shifting between several healthcare actors, including patients and their families, have an unprecedented impact on medical practice. ${ }^{6}$ Hence, there is also an explicit call for doctors' increased proficiency in multilevel 'organisational leadership': at team, organisational, system and even at societal levels. ${ }^{7-10}$

Since significant, ongoing healthcare changes cannot be achieved without their effective cooperation and support, ${ }^{11}$ doctors are progressively seen as the natural choice to lead transformation. ${ }^{12-14}$ However, the concept of 'leadership' often meets with resistance or scepticism within the (typically conservative) medical communities. ${ }^{14-16}$ Postgraduate and undergraduate medical education focus almost exclusively on clinical skills, also known as 'technical skills', while the training of 'soft skills' (like leadership skills) remains largely underrepresented. ${ }^{17}$ Despite the increasing attention on ML, the construct itself is still in its infancy when it comes to operationalising it in daily clinical practice and education. ${ }^{1418}$

Recently, the internationally leading framework on medical competencies, Canadian CanMEDS Physician Competency Framework, further propelled the importance of leadership competencies, by changing the 'manager' competency category into 'leader'. ${ }^{19}$ This concurs with numerous other efforts to develop ML development schemes, reflected also in an increase in peer-reviewed scientific reports on ML (see figure 1). Until now scholars have primarily reported on regional and organisational-level (eg, hospitals) ML development initiatives. These are essential activities operating towards hands-on acquirement of $\mathrm{ML}$ competencies by (groups of) individual doctors and often focus on the local needs and demands in improvement and innovations and habitually enhancing collaboration within interprofessional settings. ${ }^{20}$ However, ML developments at large also indicate a gradual reconstruction of professional role identity of physicians. ${ }^{21}$ Having thrived on the 'privileges of self-regulation and self-policing'22 (p.673) for centuries and positioned at the heart of the highly institutionalised healthcare industry, the strongly autonomous and prestigious medical profession is not likely to adjust to current societal and industry-specific curtails, only by means of local or organisational initiatives.

The more practical oriented ML activities executed at local, regional or organisational levels are of great importance to create change and to better understand the dynamics of role identity shifts that physicians go through. ${ }^{23}$ However, sustainable (national) reconstruction of professional identity of physicians will also require thorough '(re)rooting' at a higher, central level, 


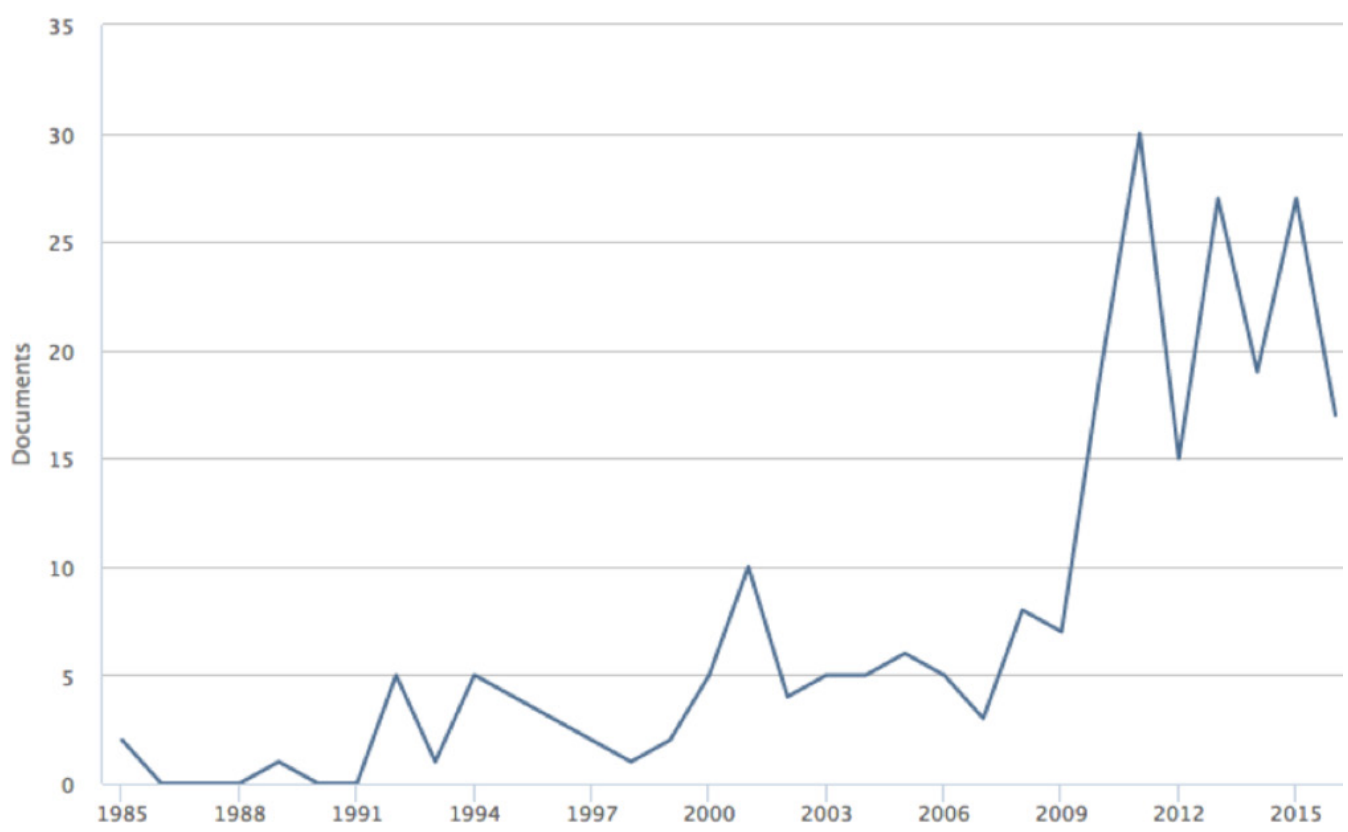

Figure 1 Search results on 'medical leadership' in Scopus electronic database from 1985 to 1 November 2016.

in particular within the context of various national medical specialists' associations and a conglomerate of inspectorates and other high-level regulating stakeholders that comprise a national healthcare system. ${ }^{24}$ It is in this context that some highlight the importance of adopting a systematic, wellarticulated approach to developing ML. ${ }^{25}$ However, despite many rich reports of ML development on regional and/or organisational levels, little is known about effective ML strategies and tactics at a national level, including the entailed ML schemes or the factors that can influence these needed developments. Yet, although mainly based on early pioneering nations, the body of knowledge on ML development is growing. The countries currently embarking on ML development lack an overview of possible (national) approaches and influencing factors in this journey. The present report on the current state of affairs is specifically for the national actors, to aid them in taking the most adequate avenues while anticipating possible barriers and to encourage them at the same time to tailor their national ML strategies to country-specific and other local needs and demands, including aligning strategies with preceding and simultaneous regional, local and institutional activities.

\section{METHODOLOGY}

We use a structured approach in this review to identify relevant publications and to extract the information from them in order

\section{Box Review methodology}

- Aims clearly identified and SPICE-based 'framing' of search strings.

- Scoping study: refining to final search strategy.

- Iterative title and abstract screening for selection.

- Forward and backward snowballing.

- Iterative data extraction and thematic coding of full-text papers.

- Consensus between researchers on themes.

- Data analysis and synthesis.

- Consensus on final outcomes. to answer the research questions (box, table 1). Our approach does not deviate from the methodology registered earlier (PROSPERO registration number: 2016:CRD42016048885).

\section{Aim and framing search}

The primary aim of this review is to systematically synthesise the existing reviews of literature that dealt with the development of ML at a national level. The secondary objectives are to answer the following questions:

- Question 1: How is ML defined?

- Question 2: How do the national ML development processes develop over time?

- Question 3: What determinants-facilitating or impedingshould be considered when developing ML at a national scale?

- Question 4: What 'interventions' have been deployed to facilitate the national development of ML?

These research questions are framed according to the SPICE principle ${ }^{26}$ into four search constructs, including related synonyms and more specific search terms (table 2). The most relevant sources for this study were selected from the electronic databases: PubMed, Scopus, Web of Science, Ovid MEDLINE and Science Direct.

The search is based on publications from the preceding 5 years following the findings of our scoping review (see below): 1 January 2011 until 15 June 2016. We chose this period because ML is a fairly new subject, and publications about ML increased rapidly from 2009 (figure 1). Based on findings during our scoping review (see below), it was decided to begin searching as of early 2011 for relevant reviews of the literature just after this steep increase.

\section{SCOPING REVIEW}

Based on our initial search strings, a scoping review ${ }^{27}$ was done to identify key articles about leadership development for healthcare professionals in general. This initial search resulted in a large number of citations $(n=20984)$ and a subsequent 'doctors only' search in 5932 results. On studying publications that were relevant to our research focus, it was decided to delete 
Table 1 Characteristics of the included review articles

\begin{tabular}{|c|c|c|c|}
\hline Author and publication year & Type of review article & $\begin{array}{l}\text { No. of } \\
\text { references }\end{array}$ & Countries \\
\hline 1. McKimm et al, $2009^{31}$ & Comparative review of ML development & 40 & UK; New Zealand \\
\hline 2.0'Sullivan and McKimm, $2011^{32}$ & Case study-based review of ML development & 16 & $\begin{array}{l}\text { Denmark; USA; Canada; Italy; } \\
\text { Australia; New Zealand }\end{array}$ \\
\hline 3. Coltart et al, 2011 & Literature search based 'viewpoint' on ML development for early career doctors & 24 & UK \\
\hline 4.Webb et al, $2014^{35}$ & Systematic literature review on medical curricula containing ML teaching interventions & 34 & Various countries \\
\hline 5. Jorm and Parker, 2014 & $\begin{array}{l}\text { Literature based 'perspective' on (lack of) evidence for ML development programmes } \\
\text { and education }\end{array}$ & 25 & Australia \\
\hline 6. Sebastian et al, 2016 & General review on a national ML framework & 65 & Australia \\
\hline 7. Hartley $2016^{36}$ & Literature review-based assessment framework for ML development across systems & 70 & Various countries \\
\hline
\end{tabular}

generic leadership development-related terms, like 'improve*, 'program(me)', 'engagement', as these terms resulted in citations not relevant to this study. Furthermore, it was determined to exclude papers on 'medical professionalism'; our focus was strictly on those reporting on ML and its national developments. Since the assessment of ML roles appeared to be an important topic, the search term 'assess"' was added. The initial searches revealed a multitude of reports on local or regional ML initiatives. It was decided to include only those studies that were related to national ML development initiatives, including those on a specific medical specialty in a country. Since our scoping search revealed that non-review-type articles often describe non-nationwide (eg, regional or local) initiatives, which are outside this study's national scope, and that review type articles often have a national scope, only articles indexed as reviews were regarded eligible for selection.

During several iterative data clinics, the researchers (MP, WK and JT) discussed the correctness of the inclusion and exclusion criteria based on a test sample of citations $(n=50)$, resulting in the fine-tuning of the inclusion and exclusion criteria. After a final revision, a second test sample of citations $(n=200)$ was used to check the adequateness of the revised criteria, using the recurrence of the earlier identified key papers as 'indicator'. The last data clinic finalised the set of inclusion and exclusion criteria and database-specific search strings (see online supplementary appendices A-D*).

\section{SELECTION PROCESS}

Records retrieved from the consulted databases were exported to Endnote to be processed for further inclusion analysis, and duplicate records were manually removed. To ensure no relevant articles were missed, forward and backward snowball techniques were used on key publications. ${ }^{28}$ This resulted in 63 (backward and forward) citations and 53 additional records.

Three researchers (MP, JT and WK) independently checked all titles and abstracts on eligibility for inclusion against the predetermined inclusion and exclusion criteria and coded them with ' 1 ' (not include), '2' (include) or ' 3 ' (potentially include: insufficient information). The inclusion process followed an iterative approach, and the researchers convened in additional data

\begin{tabular}{ll}
\hline Table 2 & Research question framed according to SPICE framework \\
\hline Setting (where?) & Various national healthcare settings \\
\hline Population (who?) & Doctors/physicians \\
\hline Interventions/determinants (what?) & Developments \\
Comparison (what else?) & (Not applicable) \\
\hline Evaluation (what results?) & Leadership \\
\hline
\end{tabular}

clinic sessions to discuss and ensure consistency and validity of the findings, resulting in conclusive selection consensus between the researchers. A fourth researcher (CW) was available for consultation if a dispute were to arise during any of the study stages, with veto privilege. However, no use was made of this decision-making process.

It was concluded that 1179 citations did not meet the predetermined inclusion criteria and were therefore excluded. From the retrieved records, 11 met the inclusion criteria and were thus included. Records that did not provide sufficient 'eligibility' information in the title or abstract were retrieved (in full text) and analysed independently by three researchers (MP, JT and WK). After reviewing the full-text versions of the initially included records, seven articles were identified as meeting the predetermined inclusion criteria (table 1). Four of the included papers originated from database searches; three resulted from the 'snowball' search. For a complete overview of this inclusion and exclusion process, see figure 2 .

\section{Data extraction}

The full-text versions of the seven included articles were then independently analysed by three researchers (WK, MP and JT). These articles were screened for: eligibility, inclusion and the compilation of an initial coding scheme, based on the 'grounded theory' of open coding principles. ${ }^{29}$ During the data extraction, themes from one or more of the specific research questions on 'definition', 'developmental processes', 'determinants' and 'interventions' were identified (see above).

The included seven articles were analysed and discussed in an iterative manner; each researcher selected relevant quotations from each paper and noted them in a table (Microsoft Word), including a short description and a suggestion for an appropriate theme ('code'). They discussed their findings during intermittent data clinics, and differences in opinion were solved through negotiated consensus. The three included 'snowball articles' were coded after the data extraction ('coding') of the initial four included articles was completed. Although the 26 themes identified in the first four articles were enriched with quotes from the 'snowball articles', the additional three papers did not provide new themes, which might indicate 'data saturation'. ${ }^{30}$ The fulltext data extraction, from the seven included articles, resulted in a 'consensus table' of quotations and themes.

In sum, our systematic literature review resulted in a list of characteristic quotes relating to the definition of 'medical leadership' (research question 1) and a 'consensus table' with quotes and short descriptions reflecting the 26 themes (research questions 3 and 4) (see online supplementary appendices E, F*). The articles included in this systematic literature review did not have 


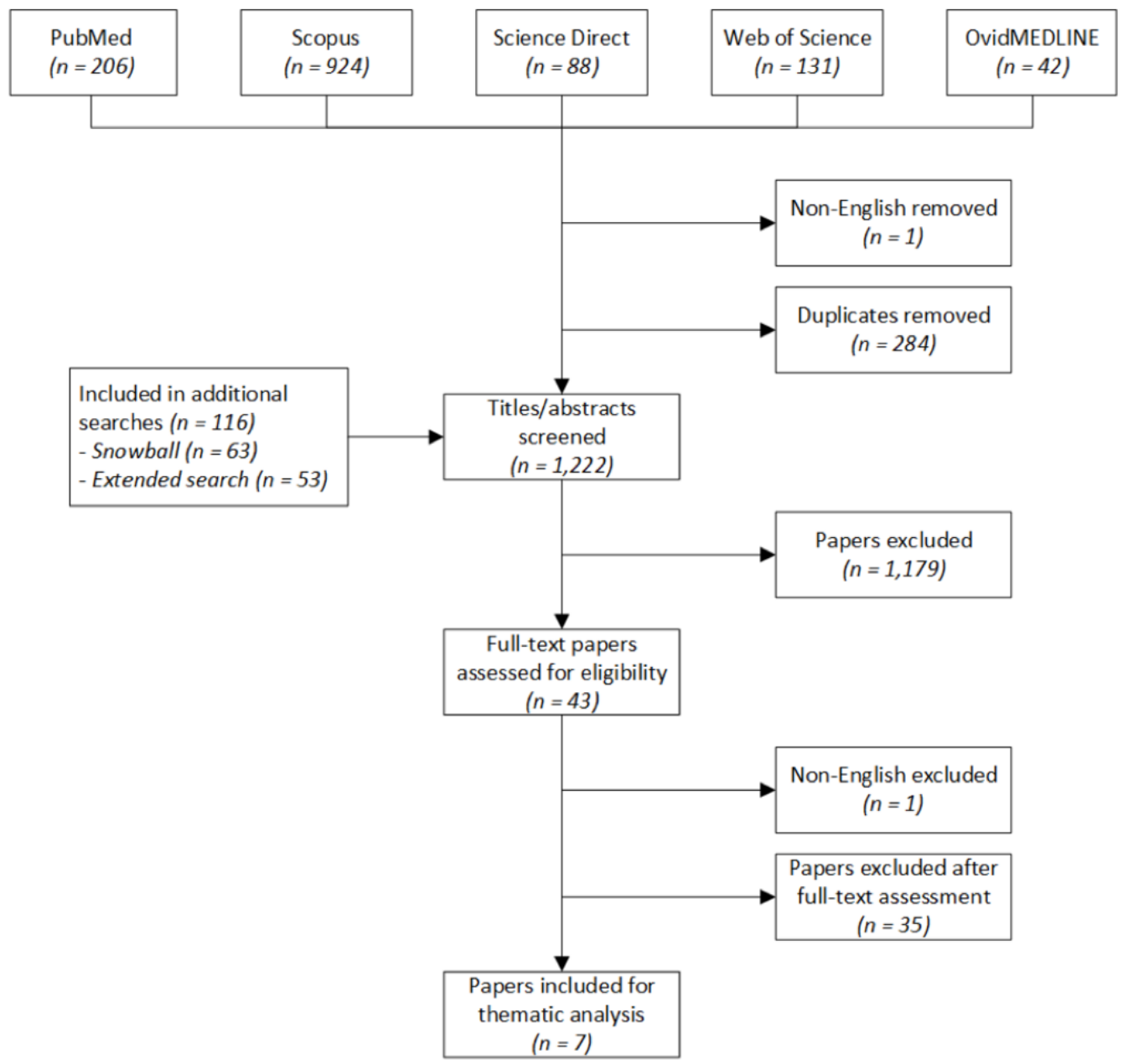

Figure 2 Diagram of the search process preceding this review.

direct references to developmental processes (research question 2), so no results are reported here for this research question.

\section{DATA SYNTHESIS}

One researcher (WK) synthesised the collected data, grouping all 26 identified themes and their descriptions into a comprehensive set of main thematic categories and subcategories. This conceptual version of a thematic table with 'determinants' and 'interventions' was then shared with the other researchers and, after providing written comments on this table (MP and JT), the researchers convened in theme clinics to discuss the correctness and phrasing of this synthesis. Subsequently, this table was revised and redrafted, and after a final reviewing round and meeting, all the researchers agreed on the two synthesised thematic tables (see table 3A; table 3B).

\section{RESULTS}

Within the scope of our search for papers on national ML development initiatives, including those within medical specialties, seven published papers report the results of various review methodologies (see table 1). Two of them discuss ML development based on case studies. ${ }^{31} 32$ One paper reports on a national ML programme $^{18}$ and one reflects on the (lack of evidence on) return on investments of a national programme. ${ }^{33}$ Two papers focus on leadership development in medical education, ${ }^{34} 35$ while the recent Hartley paper provides a framework for comparing and assessing national ML development among nations. ${ }^{36}$

\section{DEFINITION}

Some authors place ML as part of the broader 'clinical leadership' paradigm, a term that refers to all health professions. ${ }^{31}$ Although ML definitions remain unambiguous, the concept seems strongly related to doctors' positions, which in effect are influenced by a variety of factors that vary per country. Some mention a national 'style' that characterises the medical profession, for example, the 'continental' versus 'liberal' style. ${ }^{32}$ Others describe the influence of doctors' autonomy and independence on the level of their engagement in (national) administrative roles. ${ }^{34}$ However, some describe a gradual shift of doctors' positions in healthcare and society away from the area of 'power' and that the 'grooming of individuals for executive roles' is related to the (emergence of) ML. ${ }^{18}{ }^{34}$ In the latter domain, ML development programmes have more of 'a focus on developing managerial and administrative competencies, rather than a focus on leadership per se ${ }^{31}$ (p. 19). Authors mention that this shift towards (distributed and more informal) leadership is a core competency of all clinicians and that the trend is moving towards defining ML for doctors at all levels. ${ }^{33} 35$ According to the data from the studied papers, the ML definition also comprises managing change and working with other professionals. In this perspective, ML can be positioned as a social and/or societal construct, since it also entails aligning people to realise continuous improvement at all healthcare levels, for example, through facilitating group 
Table 3A Synthesis of results: determinants of national ML development

\begin{tabular}{|c|c|}
\hline (Sub)categories & Description \\
\hline I: Taxonomy & $\begin{array}{l}\text { Definition, description and } \\
\text { classification of the concept of ML and } \\
\text { its underlying principles }\end{array}$ \\
\hline 1. Meaning and focus & $\begin{array}{l}\text { Clearly defined and described meaning } \\
\text { and focus of ML during its developmental } \\
\text { phases }\end{array}$ \\
\hline 2. Diffusion of meaning and focus & $\begin{array}{l}\text { Level of (shared) understanding regarding } \\
\text { ML and its development }\end{array}$ \\
\hline II: Health system & $\begin{array}{l}\text { Combination of organisation, resources } \\
\text { financing and management }\end{array}$ \\
\hline 1. National approach & $\begin{array}{l}\text { National strategy for ML development, } \\
\text { based on systematic, system-wide, } \\
\text { interprofessional and evidence-based } \\
\text { approaches }\end{array}$ \\
\hline 2. Structural challenges & $\begin{array}{l}\text { Organisational aspects in the healthcare } \\
\text { system that can impede or facilitate ML } \\
\text { development }\end{array}$ \\
\hline
\end{tabular}

III: Cultural aspects

1. Professional culture

Characteristics and value systems of particular groups

Values, beliefs and attitudes of the medical profession, impacting their engagement in ML development

2. Societal culture

Role of public opinion and/or media in ML development

3. Recognition Recognition of ML as part of the career structure of all doctors

4. Mind set Level of interest in and attitude of doctors towards ML and its development

5. Subcultures Power balance between groups (eg, government vs medical profession; doctors vs managers)

6. Exposure Influence of doctors in key positions (eg, in national politics or management)

IV: Governance

Establishment of relevant policies and monitoring of their proper implementation

1. Political climate National political acknowledgement of the roles of doctors and the importance $\mathrm{ML}$ development

2. Regulations and rules Regulations requiring doctors to be active in management or to engage in (periodical, obligatory) ML development

V: Education

Representation of ML in undergraduate and postgraduate medical education and training

1. Alignment

Alignment of ML development curricula and training programmes across educational institutions

2. Standardisation and quality control Standardising of ML development activities, identifying best practices and monitoring outcomes

3. Longitudinal and integrated training ML development activities over extensive period of time ('cradle-to-grave') and based on career phase

4. Expertise of teachers Clearly defined expertise and requirements for instructors, trainers and educators in $\mathrm{ML}$ development

5. Partnerships

Investment in education partnerships, for example, researchers, universities and ML development providers

6. Conditions of education Conditional requirements, for example, timing, aims, duration, costs, accreditation and so on

$\mathrm{ML}$, medical leadership
Table 3B Synthesis of results: interventions facilitating national ML development

\begin{tabular}{|c|c|}
\hline Interventions & $\begin{array}{l}\text { Agents, activities or processes that can } \\
\text { facilitate ML development nationally }\end{array}$ \\
\hline $\begin{array}{l}\text { 1. National rules and } \\
\text { regulations }\end{array}$ & $\begin{array}{l}\text { Specific governmental and organisational policies } \\
\text { emboldening } M L \text { and its development }\end{array}$ \\
\hline 2. Nurturing environment & $\begin{array}{l}\text { Conditions enabling and stimulating doctors to } \\
\text { develop and deploy ML competencies in daily } \\
\text { practice }\end{array}$ \\
\hline 3. Resources & $\begin{array}{l}\text { Investments in, for example, research, conditions } \\
\text { (payed time for training and so on), high-quality } \\
\text { training materials (eg, assessments) and guidance } \\
\text { (eg, proficient trainers) }\end{array}$ \\
\hline 4. Champions & $\begin{array}{l}\text { Trusted and knowledgeable role models inspiring } \\
\text { others to engage in ML development }\end{array}$ \\
\hline 5. Professional organisations & $\begin{array}{l}\text { Professional organisations actively supporting and } \\
\text { facilitating ML development, nationally, locally and } \\
\text { specialism specific }\end{array}$ \\
\hline 6. Support & $\begin{array}{l}\text { Active supporting programmes/schemes at all levels } \\
\text { (eg, individual, at work and in specialty) to enable } \\
\text { ML development }\end{array}$ \\
\hline 7. Triggers & $\begin{array}{l}\text { Certain activities or processes that trigger ML } \\
\text { development (eg, policy change and culture shift) }\end{array}$ \\
\hline 8. Framework & $\begin{array}{l}\text { Presence, status of dissemination and quality of a } \\
\text { national ML framework }\end{array}$ \\
\hline 9. Collaboration & $\begin{array}{l}\text { Cross-sectoral and interdisciplinary collaborations } \\
\text { and partnerships in (clinical) leadership } \\
\text { development }\end{array}$ \\
\hline 10. Supportive information & $\begin{array}{l}\text { High-level administrative reports, documents and } \\
\text { scientific work presenting evidence and background } \\
\text { of } \mathrm{ML} \text { and its importance }\end{array}$ \\
\hline
\end{tabular}

$\mathrm{ML}$, medical leadership.

sense making. ${ }^{335}$ Excerpts from the studied papers relating to the various definitions of ML can be found in the online appendices (see online supplementary appendices $E^{*}$ ).

\section{DETERMINANTS}

By answering the research question on ML's determinants, the data synthesis process provides five themes of factors that facilitate or impede the national development of ML (see table 3A; table 3B).

\section{Determinant 1: taxonomy}

National ML development is propelled by a clear description, meaning and focus of the ML concept. ${ }^{35}$ Sebastian and colleagues emphasise that not having a comprehensive and collective understanding of leadership is one of the reasons that ML development 'has been problematic for those seeking to change practice ${ }^{18}$ (p.362). A compelling and widely distributed taxonomy is essential for the recognition and acknowledgement of the influence of $\mathrm{ML}$ and the behaviour of physicians on organisational effectiveness in healthcare services. Programmatic activities and well-accepted instruments, like a national ML competency framework, come into play in such circumstances. ${ }^{31}$ McKimm and colleagues argue that the Medical Leadership Competency Framework in the UK has reinforced the embedment of ML development in all UK specialty training curricula. Following the international comparative method provided by Hartley, such frameworks are seen as crucially fundamental to ML development worldwide. ${ }^{36}$ Even so, gradual shifts in meaning and focus of the ML concept during specific phases of national ML development should be considered, and it is at such times that the popular distinction between 'managerial leadership' and 'medical leadership' emerges. ${ }^{33}$ 
Determinant 2: health system

Important determinants for national ML development emanate from the method in which a national health system is structured and organised. Compared with more fragmented, local developments, a national ML development approach may be more effective in convincing and encouraging individual doctors to engage in ML. ${ }^{18}$ 31-34 Moreover, awareness and acknowledgement of the importance of ML can be enhanced by 'cradle-to-grave' programmes, providing concrete ML development opportunities throughout all medical career phases. It is suggested that such approaches can gain from leadership strategies focusing on all clinical professions. ${ }^{3135}$ Hence, ML development should be positioned as an essential element in doctors' career structures and made noticeable in medical school curricula as well as in the employment arrangements within the domain of human resources in healthcare organisations. ${ }^{32-35}$

A national process of embedding ML development within a healthcare system at large calls for active endorsement of highlevel administrators, adequate regulations, in combination with research and continuous dialogue with stakeholders. ${ }^{18} 32$ Also, contextualisation is required to meet particular needs and conditions. Geographical regions or subsystems, and also medical specialties, are characterised by specific needs and demands that require tailored approaches in order to create successful ML development schemes. ${ }^{18}$

\section{Determinant 3: cultural aspects}

A variety of cultural determinants are of importance as well. First, and most prominently, the deeply enshrined system of values and beliefs within a particular medical community must be considered. Since ML development has an impact on doctors' professional behaviours and logics, ML activities often meet with resistance. Sebastian and colleagues argue that promoting ML can be difficult due to the culture of the profession that can limit necessary innovation and change. ${ }^{18}$ Second, culture in a broader sense (eg, public opinion and influence of the press) can influence ML development. Third, significant impact is attributed to the level of recognition of ML as an integral part of daily medical practice. Lack of such recognition can fuel disinterest and avoidance behaviour among doctors towards ML and its development, resulting in significant barriers to implementation. ${ }^{33}$ A fifth subcategory reflects the effects of power (re) balances between certain autonomy subcultures and doctors' motives to engage in ML (eg, friction between management vs doctors; politics vs healthcare professionals). ${ }^{31}{ }^{36}$ Last, the positioning of doctors in specific high-exposure roles (eg, in politics and high-level healthcare administration) are acknowledged to spur national ML development. ${ }^{32}$

\section{Determinant 4: governance}

National policies that foster recognition of the importance of ML, and (peer-) encouragement of engagement in ML development, are noted as significant factors. ${ }^{34}$ Moreover, positive effects are accredited to formal regulations that require doctors to be active in management and to take part in (mandatory) ML developmental activities. Over the last decade, formal policies have emerged about the future role of doctors in leading change and innovation in health services, and numerous other practices, documents and reports have been shown to be important in stimulating ML development in several countries. ${ }^{3135}$ Hereby, local as well as national professional bodies play an important role. $^{36}$

\section{Determinant 5: education}

Most authors stress the importance of education in national ML development. Exposing early career doctors to ML development is emphasised to benefit the wider healthcare system. ${ }^{183436}$ Jorm and Parker argue that integrating a lot of leadership training into the medical curriculum is perhaps wasted because physicians often return to their daily clinical work settings where they are not encouraged or enabled to employ their new set of skills. ${ }^{33}$ However, ML training 'drip fed' throughout the duration of medical education'36 (p.36) could prevent such a 'wash-out' effect. However, training in stages is not the only factor to be considered. McKimm and colleagues emphasise the challenge of identifying the educators in ML. ${ }^{31}$ The work of ML trainers and teachers should be continuously evaluated according to well-founded rigorous theoretical bases and best practices that are proven to be effective in their appropriate context. Hence, specifying effective requirements for ML education are essential when developing, deploying and evaluating effective ML programmes, and this includes a contingent of proficient educationalists. ${ }^{36}$ Aligning leadership curricula with existing competency frameworks would create opportunities to standardise particular learning activities and assessment methods and to enable the comparing of the outcomes of such new elements of medical education. ${ }^{1835}$

\section{Interventions}

Methods to enhance or facilitate ML development at the national level varied from: the development of a national ML competency framework (see above) to the identification and facilitation of champions (see table $3 \mathrm{~A}$; table $3 \mathrm{~B}$ ). ${ }^{33}$ Such methods exist in the form of agents, processes or practical activities that can be deployed formally or informally to promote ML development. Many authors stress the importance of substantial investments and resources, also in terms of time, at all career phases, to enable doctors to engage in a great variety of ML development activities. $^{313235}$

\section{LIMITATIONS}

Besides the strength of now having an overview of the various national ML developments worldwide, this study has limitations. The large number and variety of published ML-related papers and the absence of an internationally recognised taxonomy of the concept of ML might have influenced the accurateness of our search strategy, possibly resulting in missing papers that are indexed with different search terms. Moreover, our data extraction might have been biased because the ML literature offers a wide diversity of ideas and concepts related to ML and its national development. This is undisputedly a characteristic of any review examining a fairly new, multifaceted and context-sensitive construct like ML. Also, regarding the young age of the ML field, as well as the fact that our methodology did not consider 'grey literature', many more insights and experiences might not have been captured here. Limiting our search to the English language may have led to a cultural as well as a language bias. Finally, the decision to focus primarily on review type articles could have led to a selection bias. However, the young age of the concept of ML, as well as the intensive iterative snow-ball approach that was applied in our search, in our opinion provides confidence in having captured most, if not all, relevant publications to date.

\section{DISCUSSION}

We undertook this review to search in order to synthesise the existing reviews on ML development in a national context. 
Every single studied paper stressed the challenges that must be overcome to increase ML awareness of doctors, medical trainees and students. Doctors may need to engage unequivocally in small and large initiatives to develop leadership competencies to maintain their crucial roles and positions within the increasingly complex 'networks' in which they function. ${ }^{31}$

We found only few reports studying ML development from a national-level perspective. Scientific reports on ML and its development for particular medical specialties also do not reflect from a national implementation perspective. None of the reviewed papers provide detailed insight into processes, stages or phases that can characterise the evolvement of national ML development (research question 2). We believe that this indicates that ML development is still in a relatively early phase and various nations as well as medical specialties will increasingly embark on organising ML development over time. This is also since, in our opinion, it requires time for national ML development to be 'translated' adequately to specific medical specialties in a country.

Our data suggest that deploying ML development at a national level should be 'multi-faceted', based on a bundle of different interventions and strategies. Additionally, selection of adequate strategies, tactics and interventions as well as their planning and deployment should, in all cases, be tailored to the given national context.

Like all changes and innovations in medicine, the level of evidence base for new educational interventions, in our case ML training and education, must be taken extremely seriously to convince physicians of the added value. ${ }^{18}$ As with many other professionals, doctors should be regarded as a group of highly academically inclined, busy professionals that are quite critical about sensitive topics like the development of their professional identity. ${ }^{37} 38$ At this point, the effectiveness of possible approaches and interventions used to facilitate doctors in ML development (as part of their medical responsibilities), remains largely unknown. Also, validated assessment methods for measuring and monitoring the development of ML competencies have not been defined well yet, and several scholars mention the necessity of empirical evidence for the value of ML development, for example, in terms clearly defined return-on-investment principles. ${ }^{1831}$

Some scholars specify that ML development at all career levels should be based on interprofessional perspectives that increasingly characterise modern clinical practices. ${ }^{31} 34$ Coltart et al suggest that 'the strong challenge to the medical profession internationally is to move beyond traditional notions of hierarchy and leadership from an elite minority, and begin investing in the leadership attributes of all its future workforce ${ }^{34}$ (p. 1849).

The data presented in our study emphasise that the reinstitutionalisation of the essential position of doctors, who often bear the highest responsibilities within the care processes of patients, and enabling them to act accordingly, is a complex and non-linear process. ${ }^{38}$ In our view, effective management is based on good leadership. Hence, 'effective leadership' of doctors is a qualification that is worth pursuing. Leadership scholars and practitioners may be of help for this cross-disciplinary endeavour. Further scientific scrutiny is needed to chart the consequences of these relatively new institutional dynamics, including all sorts of cultural shifts and changes in professional roles and identities that result from healthcare system transformations currently taking place across the world. ${ }^{20}$ Currently, only six countries worldwide (Australia, Canada, Denmark, the Netherlands, New Zealand and the UK) have established a certain level of national ML development. Further research on the experiences from these 'trail-blazing' countries could be advantageous for other nations that are novices in this field. Such studies could reveal more in-depth insight in the effectiveness of ML development interventions, in particular when combining and aligning local, institutional and national agency in the perspective of healthcare transformation.

\section{CONCLUSIONS}

To the best of our knowledge this is the first systematic review of the literature on ML development with a national perspective. Our study indicates that national healthcare transformation processes involve a rethinking and remaking of some of the oldest institutionalised logics within these systems and their clinical subcultures: the role and position of medical doctors. This includes the need to extend the behavioural repertoire of doctors: to enable them to play a key role in improving and innovating the processes of their own work. Over the years, the requirements for an adequate skill set of doctors have been altered, particularly relating to behavioural and 'managerial' competencies. $^{2037}$ In our study, the reviewed ML authors share the insight that more doctors should become involved in these increasingly dynamic processes.

The data we present here provide nations that are contemplating ML development with some initial guidance, based on specific determinants and interventions used in countries that are currently involved in these activities. Our research also suggests that any national approaches aimed at meeting the challenges of engaging doctors in system transformation should be robust, multifaceted, intensely endorsed and comprehensively resourced in order to bear any fruit. In particular, we think that current literature and experiences indicate that national implementation of ML development has to be well tailored and well deployed. Also, in our opinion, the studied literature convincingly shows the importance of investing in ML education across all levels and all phases of doctors' careers.

Our study also demonstrates that developing leadership competencies for doctors faces the myriad of perspectives that typify healthcare system transformations. Effective ML development seems, therefore, to be a part of larger scale changes and is far from 'just another training'. Moreover, sociology, organisational behaviour, education, public economics, administration and governance are all relevant fields that should be considered when studying the so-called normalisation processes underlying ML development at a national level. These scientific efforts can aid to better understand effective change and innovation in healthcare. They should comprise in-depth scrutinising ML 'identity work' at regional, organisational, as well as at individual level, considering both the dynamics of institutionalisation and professionalisation. ${ }^{39}$

In the face of the numerous organisational and local activities, a focus on the national perspectives of ML is also of relevance given that ML might evolve with a certain stratification. A distinction can be made between vertical and horizontal levels of ML. Medical executives and other managers, frontline medical innovation and quality 'champions' and physicians performing based on 'regular' day-to-day clinical leadership encompass a vertical classification. However, the diversity between medical specialisations can bring about a variety of (horizontal) ML types, characterised by specific activities and roles of physicians in a certain medical specialty. For example, although both should be able to display 'regular' ML competencies, work and work setting of a family physician and a surgeon can demand different leadership skills and styles. Finally, also 
because ML competencies focus particularly on interdisciplinary collaboration, it is relevant to consider intertwinement of leadership education and training for the healthcare workforce at large. Defining and implementation of such stratification principles of medical and clinical leadership can prosper from effective national level coordination and collaboration between related professional associations and other stakeholders.

The myriad of multilevel factors that influence professional role identity (re)construction are well documented. These include 'macro' level institutional activities coming from governmental and health authorities, professional organisations, overarching regulations and (other) social, societal and economic factors that impact jurisdiction of physicians. ${ }^{40}$ Building on these actors and related factors and on the experiences of trailblazing countries in national ML development strategies, we hope to contribute to more in-depth understanding of the institutional forces that are to be taken into consideration here. We hope that our work adds to an ongoing cross-disciplinary and international movement, which enables (aspiring) doctors (and others) to develop adequate 21st-century competencies that include effective ML. This is because if effective ML is not taught, ineffective ML may become the undesirable standard.

Contributors All authors contributed to the development of this paper, and all were involved in the drafting and editing of the manuscript and final approval.

Competing interests None declared.

Provenance and peer review Not commissioned; externally peer reviewed.

Data sharing statement Online appendices to this article are available online: https://www.researchgate.net/publication/319711231_A_Review_of_ Determinants_of_National_Medical_Leadership_Development_-_BMJ-L.

(c) Article author(s) (or their employer(s) unless otherwise stated in the text of the article) [year]. All rights reserved. No commercial use is permitted unless otherwise expressly granted.

\section{REFERENCES}

1 Cochran J, Kaplan GS, Nesse RE. Physician leadership in changing times. Healthc 2014;2:19-21.

2 Keijser W, Smits J, Penterman L, et al. Physician leadership in e-health? A systematic literature review. Leadersh Health Serv 2016;29:331-47.

3 West M, Loewenthal L, Armit K, et al. Leadership and leadership development in health care: The evidence base. London: Faculty of Medical Leadership and Management, 2015. https://www.kingsfund.org.uk/sites/files/kf/field/field_ publication_file/leadership-leadership-development-health-care-feb-2015.pdf (accessed Apr 2017).

4 Berwick D. A promise to learn-a commitment to act: improving the safety of patients in England. London: Department of Health 2013;6.

5 Spurgeon P, Clark J, Ham C. Medical leadership: from the dark side to centre stage. London: Radcliffe Publishing Ltd, 2011.

6 Keijser W, de Manuel-Keenoy E, d'Angelantonio M, et al. DG connect funded projects on information and communication technologies (ict) for old age people: beyond silos, carewell and smartcare. J Nutr Health Aging 2016;20:1024-33.

7 Chadi N. Medical leadership: doctors at the helm of change. Mcgill J Med 2009;12:52-7.

8 Reinertsen JL, Gosfield AG, Rupp W, et al. Engaging physicians in a shared quality agenda. Cambridge, MA: Institute for Healthcare Improvement, 2007. http://www. reinertsengroup.com/publications/documents/IHIEngagingPhysiciansWhitePaper2007. pdf (accessed Apr 2017).

9 Keijser W. Teamwork, leadership, and continuous improvement. In: Glaudemans A, Medema J, Van Zanten AK, eds. Quality in nuclear medicine. Basel: Springer International Publishing, 2017:323-9.

10 Janss $R$, Rispens $S$, Segers $M$, et al. What is happening under the surface? Power, conflict and the performance of medical teams. Med Educ 2012;46:838-49.

11 Bowns I, McNulty T. Re-engineering leicester royal infirmary: An independent evaluation of implementation and impact. Sheffield: School of Health and Related Research, University of Sheffield, 1999.
12 Chan MK, de Camps Meschino D, Dath D, et al. Collaborating internationally on physician leadership development: why now? Leadersh Health Serv 2016;29:231-9.

13 Clark J. Medical leadership and engagement: no longer an optional extra. J Health Organ Manag 2012;26:437-43.

14 Bohmer R. The instrumental value of medical leadership. Engaging doctors in improving services. London: The Kings Fund, 2012. https://www.kingsfund.org. uk/sites/files/kf/instrumental-value-medical-leadership-richard-bohmer-leadershipreview2012-paper.pdf (accessed Apr 2017).

15 Ham C. Doctors in leadership: learning from international experience. The International Journal of Clinical Leadership 2008;16:11-16.

16 Kyratsis Y, Armit K, Zyada A, et al. Medical leadership and management in the United Kingdom. Australas Psychiatry 2016;24:240-2.

17 Warren 0J, Carnall R. Medical leadership: why it's important, what is required, and how we develop it. Postgrad Med J 2011;87:27-32.

18 Sebastian A, Fulop L, Dadich A, et al. Health LEADS Australia and implications for medical leadership. Leadersh Health Serv 2014;27:355-70.

19 Dath D, Chan M, CanMEDS AC. From manager to leader. Ottowa: The Royal College of Physicians and Surgeons of Canada, 2015. http://www.royalcollege.ca/portal/page/ portal/rc/common/documents/canmeds/framework/canmeds2015_manager_to_ leader_e.pdf

20 Noordegraaf M, Schneider MME, Van Rensen ELJ, et al. Cultural complementarity: Reshaping professional and organizational logics in developing frontline medical leadership. Public Management Review 2016;18:1111-37.

21 Hoff T, Pohl H. Not our parent's profession. The restratification of medicine in the United States. In: Hoff T, Sutcliffe K, Young G, The healthcare professional workforce. Understanding human capital in a changing industry. New York: Oxford University Press, 2017:23-46.

22 Sullivan WM. Medicine under threat: professionalism and professional identity. CMAJ 2000;162:673-5.

23 Reay T, Goodrick E, Waldorff SB, et al. Getting leopards to change their spots: Cocreating a new professional role identity. Acad Manage J 2017;60:1043-70.

24 Greenwood R, Hinings CR, Suddaby R. Theorizing change: The role of professional associations in the transformation of institutionalized fields. Acad Manage $J$ 2002;45:58-80.

25 Ham C, Clark J, Spurgeon P, et al. Medical chief executives in the NHS. London: NHS Institute for Innovation and Improvement and the Academy of Medical Royal Colleges, 2010. http://www.birmingham.ac.uk/Documents/college-social-sciences/social-policy/ HSMC/publications/2010/Medical-Chief-Executives-in-the-NHS.pdf (accessed Apr 2017).

26 Booth A. "Brimful of STARLITE": toward standards for reporting literature searches. J Med Libr Assoc 2006;94:421.

27 Mays N, Roberts E, Popay J. Synthesising research evidence. In: Fulop N, Allen P, Clarke A, Black N, et al, eds. Studying the organisation and delivery of health services: Research methods. London: Routledge, 2001:188-219.

28 Greenhalgh T, Peacock R. Effectiveness and efficiency of search methods in systematic reviews of complex evidence: audit of primary sources. BMJ 2005;331:1064-5.

29 Wolfswinkel JF, Furtmueller E, Wilderom CPM. Using grounded theory as a method for rigorously reviewing literature. Eur J Inf Syst 2013;22:45-55.

30 Dixon-Woods M, Bonas S, Booth A, et al. How can systematic reviews incorporate qualitative research? A critical perspective. Qualitative Research 2006;6:27-44.

31 McKimm J, Rankin D, Poole P, et al. Developing medical leadership: a comparative review of approaches in the UK and New Zealand. International Journal of Leadership in Public Services 2009:5:10-24.

32 O'Sullivan H, McKimm J. Medical leadership: an international perspective. Br J Hosp Med 2011;72:638-41.

33 Jorm C, Parker M. Medical leadership is the new black: or is it? Aust Health Rev 2015:39:217-9.

34 Coltart CE, Cheung R, Ardolino A, et al. Leadership development for early career doctors. Lancet 2012:379:1847-9.

35 Webb AM, Tsipis NE, McClellan TR, et al. A first step toward understanding best practices in leadership training in undergraduate medical education: a systematic review. Acad Med 2014;89:1563-70.

36 Hartley K. Untangling approaches to management and leadership across systems of medical education. BMC Health Serv Res 2016;16:180.

37 Mcgivern G, Currie G, Ferlie E, et al. Hybrid manager-professionals' identity work: The maintenance and hybridization of medical professionalism in managerial contexts. Public Adm 2015;93:412-32.

38 Kyratsis $Y$, Atun R, Phillips N, et al. Health systems in transition: Professional identity work in the context of shifting institutional logics. Acad Manage J 2017;60:610-41.

39 Reay T, Goodrick E, Hinings R. Institutionalization and Professionalization. In: Ferlie E, Montgomery K, Reff Pedersen A, The Oxford Handbook of Health care Management. New York: Oxford University Press, 2016:25-44.

40 Chreim S, Williams BE, Hinings CR. Interlevel influences on the reconstruction of professional role identity. Acad Manage J 2007;50:1515-39. 